\title{
COMMUTANT LIFTING, TENSOR ALGEBRAS, AND FUNCTIONAL CALCULUS
}

\author{
GELU POPESCU \\ Division of Mathematics and Statistics, The University of Texas at San Antonio, \\ San Antonio, TX 78249, USA (gpopescu@math.utsa.edu)
}

(Received 20 October 1998)

\begin{abstract}
A non-commutative multivariable analogue of Parrott's generalization of the Sz.-Nagy-Foiaş commutant lifting theorem is obtained. This yields Tomita-type commutant results and interpolation theorems (e.g. Sarason, Nevanlinna-Pick, Carathéodory) for $F_{n}^{\infty} \bar{\otimes} \mathcal{M}$, the weakly-closed algebra generated by the spatial tensor product of the non-commutative analytic Toeplitz algebra $F_{n}^{\infty}$ and an arbitrary von Neumann algebra $\mathcal{M}$. In particular, we obtain interpolation theorems for bounded analytic functions from the open unit ball of $\mathbb{C}^{n}$ into a von Neumann algebra.

A variant of the non-commutative Poisson transform is used to extend the von Neumann inequality to tensor algebras, and to provide a generalization of the functional calculus for contractive sequences of operators on Hilbert spaces. Commutative versions of these results are also considered.
\end{abstract}

Keywords: commutant lifting; tensor algebras; functional calculus; interpolation

AMS 2000 Mathematics subject classification: Primary 47L25; 47A57; 47A60

Secondary 30E05

\section{Introduction}

Let $F^{2}\left(H_{n}\right)=\mathbb{C} 1 \oplus \oplus_{m \geqslant 1} H_{n}^{\otimes m}$ be the full Fock space on $n$ generators, where $H_{n}$ is an $n$-dimensional complex Hilbert space with orthonormal basis $\left\{e_{1}, e_{2}, \ldots, e_{n}\right\}$ if $n$ is finite, and $\left\{e_{1}, e_{2}, \ldots\right\}$ if $n=\infty$. For each $i=1,2, \ldots$, define the left creation operator by $S_{i} \xi:=e_{i} \otimes \xi, \xi \in F^{2}\left(H_{n}\right)$. We shall denote by $\mathcal{P}$ the set of all $p \in F^{2}\left(H_{n}\right)$ which are finite sums of tensor monomials. Define $F_{n}^{\infty}$ as the set of all $g \in F^{2}\left(H_{n}\right)$ such that

$$
\|g\|_{\infty}:=\sup \left\{\|g \otimes p\|_{F^{2}\left(H_{n}\right)}: p \in \mathcal{P},\|p\|_{F^{2}\left(H_{n}\right)} \leqslant 1\right\}<\infty .
$$

We denote by $\mathcal{A}_{n}$ the closure of $\mathcal{P}$ in $\left(F_{n}^{\infty},\|\cdot\|_{\infty}\right)$. The Banach algebra $F_{n}^{\infty}$ (respectively, $\left.\mathcal{A}_{n}\right)$ can be viewed as a non-commutative analogue of the Hardy space $H^{\infty}(\mathbb{D})$ (respectively, disc algebra $A(\mathbb{D})$ ); when $n=1$ they coincide.

In $\left[\mathbf{2 0}\right.$, Theorem 3.1] we proved that $\mathcal{A}_{n}$ is completely isometrically isomorphic to the norm-closed algebra generated by any sequence $V_{1}, \ldots, V_{n}$ of isometries with $V_{1} V_{1}^{*}+\cdots+$ $V_{n} V_{n}^{*} \leqslant I$, and the identity. It follows from [18, Theorem 4.3] that the non-commutative analytic Toeplitz algebra $F_{n}^{\infty}$ can be identified with the weakly-closed (WOT-closed) 
algebra generated by the left creation operators $S_{1}, \ldots, S_{n}$, and the identity. The algebras $F_{n}^{\infty}$ and $\mathcal{A}_{n}$ were introduced by the author in $[\mathbf{1 6}]$ in connection with a non-commutative von Neumann inequality, and have been studied in several papers $[\mathbf{2}, \mathbf{1 5}, \mathbf{1 8}-\mathbf{2 0}, \mathbf{2 2}]$, and recently in $[\mathbf{3}, \mathbf{6}-\mathbf{8}, \mathbf{2 1}]$.

We established a strong connection between the algebra $F_{n}^{\infty}$ and the function theory on the open unit ball $\mathbb{B}_{n}$ of $\mathbb{C}^{n}$ through the non-commutative von Neumann inequality [16] (see also $[\mathbf{1 8}, \mathbf{2 0}, \mathbf{2 2}])$. In particular, we proved that there is a completely contractive homomorphism

$$
\Phi: F_{n}^{\infty} \rightarrow H^{\infty}\left(\mathbb{B}_{n}\right), \quad f\left(S_{1}, \ldots, S_{n}\right) \mapsto f\left(\lambda_{1}, \ldots, \lambda_{n}\right),
$$

where $\left(\lambda_{1}, \ldots, \lambda_{n}\right) \in \mathbb{B}_{n}$. A characterization of the analytic functions in the range of the map $\Phi$ was obtained in [3,7]. Arveson proved that $\Phi$ is not surjective [4] and the functions in its range are the multipliers of a certain function Hilbert space. In $[\mathbf{3}, \mathbf{7}]$, it was proved that $F_{n}^{\infty} / \operatorname{ker} \Phi$ is an operator algebra which can be identified with $\mathcal{W}_{n}^{\infty}:=\left.P_{F_{s}^{2}} F_{n}^{\infty}\right|_{F_{s}^{2}}$, the compression to the symmetric Fock space $F_{s}^{2} \subseteq F^{2}\left(H_{n}\right)$.

In $[\mathbf{1}, \mathbf{3}, \mathbf{4}, \mathbf{7}, \mathbf{2 2}]$, a good case is made that the appropriate commutative multivariable analogue of $H^{\infty}(\mathbb{D})$ is the algebra $\mathcal{W}_{n}^{\infty}$, which is the WOT-closed algebra generated by $B_{i}:=\left.P_{F_{s}^{2}} S_{i}\right|_{F_{s}^{2}}, i=1, \ldots, n$, and the identity. In this paper, we provide further evidence that $F_{n}^{\infty}$ (respectively, $\mathcal{W}_{n}^{\infty}$ ) is a non-commutative (respectively, commutative) multivariate analogue of $H^{\infty}(\mathbb{D})$.

The main result of $\S 2$ (see Theorem 2.1) is a non-commutative multivariable analogue of Parrott's generalization [12] of the Sz.-Nagy-Foias commutant lifting theorem [24] (see also [9]). We also identify (see Theorem 2.3) the commutant of sets of the form $F_{n}^{\infty} \otimes \mathcal{S}$, where $\mathcal{S}$ is a subset of $B(\mathcal{H})$, the algebra of bounded linear operators on a Hilbert space $\mathcal{H}$, and contains the identity operator. These results are used to extend Sarason's interpolation result $[\mathbf{2 3}]$ to $F_{n}^{\infty} \otimes \mathcal{M}$, where $\mathcal{M} \subseteq B(\mathcal{H})$ is a self-adjoint set containing the identity (see Theorem 2.4). This will lead to Tomita-type commutant results. In particular, Corollary 2.6 shows that if $J$ is a WOT-closed two-sided ideal in $F_{n}^{\infty}$, then

$$
\left[\left(F_{n}^{\infty} / J\right) \otimes \mathcal{M}\right]^{\prime}=\left(F_{n}^{\infty} / J\right)^{\prime} \bar{\otimes} \mathcal{M}^{\prime}
$$

where the prime denotes the commutant and $\mathcal{A} \bar{\otimes} \mathcal{B}$ denotes the WOT-closed algebra generated by the spatial tensor product of the two algebras. Another consequence of Theorem 2.4 is a Nevanlinna-Pick type interpolation theorem for analytic functions from the unit ball of $\mathbb{C}^{n}$ into a von Neumann algebra $\mathcal{M}$, which extends results from $[\mathbf{3}, \mathbf{7}$, $\mathbf{1 1}, \mathbf{1 3}, \mathbf{2 1}, \mathbf{2 3}$. On the other hand, the non-commutative Carathéodory interpolation problem [19, Corollary 4.4] is extended to $F_{n}^{\infty} \bar{\otimes} \mathcal{M}$.

In $\S 3$, we consider a variant of the non-commutative Poisson transform introduced in $[\mathbf{2 2}]$ and provide extensions of the von Neumann type inequalities from $[\mathbf{4}, \mathbf{1 2}, \mathbf{1 6}, \mathbf{2 0}$, $\mathbf{2 2}, \mathbf{2 6}]$. This will lead to a generalization of the $F_{n}^{\infty}$-functional calculus for contractive sequences of class $C_{0}[\mathbf{1 8}]$, which also extends the Sz--Nagy-Foiaş $H^{\infty}$-functional calculus for $C .0$-contractions $[\mathbf{2 5}]$.

More precisely, let $\mathbb{F}_{n}^{+}$be the unital free semigroup on $n$ generators $s_{1}, \ldots, s_{n}$, and let $e$ be its neutral element. For any $\sigma:=s_{i_{1}} \cdots s_{i_{k}} \in \mathbb{F}_{n}^{+}$we define its length $|\sigma|:=k$, and 
$|e|=0$. On the other hand, if $T_{i} \in B(\mathcal{H}), i=1, \ldots, n$, we denote $T_{\sigma}:=T_{i_{1}} \cdots T_{i_{k}}$ and $T_{e}:=I_{\mathcal{H}}$. We show that if $\mathcal{T}:=\left[T_{1}, \ldots, T_{n}\right]$ is $C_{0}$-row contraction (see $\S 3$ for terminology) and $\Delta:=I_{\mathcal{H}}-\sum_{i=1}^{n} T_{i} T_{i}^{*}$, then every $f \in F_{n}^{\infty} \bar{\otimes}\left\{T_{1}, \ldots, T_{n}, \Delta\right\}^{\prime}$ has a unique Fourier expansion $f \sim \sum_{\alpha \in \mathbb{F}_{n}^{+}} S_{\alpha} \otimes W_{(\alpha)}$ with $W_{(\alpha)} \in\left\{T_{1}, \ldots, T_{n}, \Delta\right\}^{\prime}$, and the map

$$
f \mapsto f\left(T_{1}, \ldots, T_{n}\right):=\text { SOT- } \lim _{r \rightarrow 1} \sum_{\alpha \in \mathbb{F}_{n}^{+}} r^{|\alpha|} W_{(\alpha)} T_{\alpha}
$$

is a WOT-continuous, completely contractive homomorphism from the algebra $F_{n}^{\infty} \bar{\otimes}$ $\left\{T_{1}, \ldots, T_{n}, \Delta\right\}^{\prime}$ to $B(\mathcal{H})$. A commutative version of this result is also obtained. We mention that all the results of this paper are true if $n=\infty$, in a slightly adapted version.

After this paper was submitted for publication, Muhly and Solel published a paper [10] which contains a multivariable commutant lifting result (see their Theorem 4.4). Their result is close to our Theorem 2.1, but seems to be different. In any case, our proof is different, and is based on the geometric structure of the non-commutative minimal isometric dilation [14] and Parrott's Lemma [12].

\section{Commutant lifting, tensor algebras, and interpolation}

Let us recall from $[\mathbf{1 4}, \mathbf{1 5}, \mathbf{1 7}]$ a few results concerning the non-commutative dilation theory for $n$-tuples of operators. A sequence of operators $\mathcal{T}:=\left[T_{1}, \ldots, T_{n}\right], T_{i} \in B(\mathcal{H})$, $i=1, \ldots, n$, is called contractive (or row contraction) if $T_{1} T_{1}^{*}+\cdots+T_{n} T_{n}^{*} \leqslant I_{\mathcal{H}}$. We say that a sequence of isometries $\mathcal{V}:=\left[V_{1}, \ldots, V_{n}\right]$ on a Hilbert space $\mathcal{K} \supseteq \mathcal{H}$ is a minimal isometric dilation of $\mathcal{T}$ if the following properties are satisfied:

(i) $V_{1} V_{1}^{*}+\cdots+V_{n} V_{n}^{*} \leqslant I_{\mathcal{K}}$;

(ii) $\left.V_{i}^{*}\right|_{\mathcal{H}}=T_{i}^{*}, i=1, \ldots, n$;

(iii) $\mathcal{K}=\bigvee_{\alpha \in \mathbb{F}_{n}^{+}} V_{\alpha} \mathcal{H}$.

The minimal isometric dilation of $\mathcal{T}$ is uniquely determined up to an isomorphism. Let us consider a realization of it on Fock spaces. As in [14], let us define $D_{\mathcal{T}}: \oplus_{j=1}^{n} \mathcal{H} \rightarrow \oplus_{j=1}^{n} \mathcal{H}$ by $D_{\mathcal{T}}:=\left(I_{\oplus_{j=1}^{n}} \mathcal{H}-\mathcal{T}^{*} \mathcal{T}\right)^{1 / 2}$, and set $\mathcal{D}:=\overline{D_{\mathcal{T}}\left(\oplus_{j=1}^{n} \mathcal{H}\right)}$. Let $D_{i}: \mathcal{H} \rightarrow F^{2}\left(H_{n}\right) \otimes \mathcal{D}$ be defined by

$$
D_{i} h:=1 \otimes D_{\mathcal{T}}(\underbrace{0, \ldots, 0}_{i-1 \text { times }}, h, 0, \ldots, 0) \oplus 0 \oplus 0 \cdots .
$$

Consider the Hilbert space $\mathcal{K}:=\mathcal{H} \oplus\left(F^{2}\left(H_{n}\right) \otimes \mathcal{D}\right)$ and define $V_{i}: \mathcal{K} \rightarrow \mathcal{K}$ by

$$
V_{i}(h \oplus(\xi \otimes d)):=T_{i} h \oplus\left(D_{i} h+\left(S_{i} \otimes I_{\mathcal{D}}\right)(\xi \otimes d)\right)
$$

for any $h \in \mathcal{H}, \xi \in F^{2}\left(H_{n}\right), d \in \mathcal{D}$. Notice that

$$
V_{i}=\left[\begin{array}{cc}
T_{i} & 0 \\
D_{i} & S_{i} \otimes I_{\mathcal{D}}
\end{array}\right]
$$


with respect to the decomposition $\mathcal{K}=\mathcal{H} \oplus\left(F^{2}\left(H_{n}\right) \otimes \mathcal{D}\right)$. It was proved in [14] that the sequence $\mathcal{V}:=\left[V_{1}, \ldots, V_{n}\right]$ is the minimal isometric dilation of $\mathcal{T}$. Let $\mathcal{H}_{0}:=\mathcal{H}$ and

$$
\mathcal{H}_{k}:=\mathcal{H}_{k-1} \bigvee\left(\bigvee_{|\alpha|=1} V_{\alpha} \mathcal{H}_{k-1}\right) \quad \text { if } k \geqslant 2
$$

Notice that $\mathcal{K}=\bigvee_{k=0}^{\infty} \mathcal{H}_{k}, \mathcal{H}_{k} \subset \mathcal{H}_{k+1}$, and all subspaces $\mathcal{H}_{k}$ are invariant to each $V_{i}^{*}$, $i=1, \ldots, n$. On the other hand, we have $\mathcal{H}_{1}=\mathcal{H} \oplus \mathcal{D}$ and

$$
\mathcal{H}_{k}=\mathcal{H} \oplus \bigoplus_{|\alpha| \leqslant k-1} e_{\alpha} \otimes \mathcal{D} \quad \text { if } k \geqslant 2
$$

where $\left\{e_{\alpha}\right\}_{\alpha \in \mathbb{F}_{n}^{+}}$is the canonical basis of $F^{2}\left(H_{n}\right)$ generated by $e_{1}, \ldots, e_{n}$, i.e. $e_{\alpha}:=$ $e_{i_{1}} \otimes \cdots \otimes e_{i_{k}}$ if $\alpha:=s_{i_{1}} \cdots s_{i_{k}} \in \mathbb{F}_{n}^{+}$, and $e_{\alpha}=1$ if $\alpha=e$. Denote $\mathcal{V}_{0}:=\mathcal{T}$ and $\mathcal{V}_{k}:=$ $\left[V_{1, k}, \ldots, V_{n, k}\right]$ if $k \geqslant 1$, where $V_{i, k}:=\left.P_{\mathcal{H}_{k}} V_{i}\right|_{\mathcal{H}_{k}}, i=1, \ldots, n$, and $P_{\mathcal{H}_{k}}$ is the orthogonal projection from $\mathcal{K}$ onto $\mathcal{H}_{k}$. Notice that $V_{i, k}, i=1, \ldots, n$, are partial isometries with orthogonal final spaces and initial space $\mathcal{H}_{k-1}$. It is easy to see that $\mathcal{V}$ is also the minimal isometric dilation of $\mathcal{V}_{k},\left.V_{i}^{*}\right|_{\mathcal{H}_{k}}=V_{i, k}^{*}$, and $V_{i}^{*}=\mathrm{SOT}-\lim _{k \rightarrow \infty} V_{i, k}^{*} P_{\mathcal{H}_{k}}$.

On the other hand, let us mention that $\mathcal{V}_{k+1}$ is the one-step dilation of $\mathcal{V}_{k}$, i.e. $\mathcal{H}_{k+1}=$ $\mathcal{H}_{k} \oplus D_{\mathcal{V}_{k}}\left(\oplus_{j=1}^{n} \mathcal{H}_{k}\right)$, and, for each $i=1, \ldots, n$,

$$
V_{i, k+1}(x \oplus y)=V_{i, k} x \oplus D_{\mathcal{V}_{k}}(\underbrace{0, \ldots, 0}_{i-1 \text { times }}, x, 0, \ldots, 0)
$$

for any $x \in \mathcal{H}_{k}$ and $y \in D_{\mathcal{V}_{k}}\left(\oplus_{j=1}^{n} \mathcal{H}_{k}\right)$. Given $A \in B(\mathcal{H})$ with $A \in C^{*}\left(T_{1}, \ldots, T_{n}\right)^{\prime}$, the commutant of the $C^{*}$-algebra generated by $T_{1}, \ldots, T_{n}$, there exists a unique extension $\tilde{A}$ of $A$ to the Hilbert space $\mathcal{K}=\mathcal{H} \oplus\left(F^{2}\left(H_{n}\right) \otimes \mathcal{D}\right)$ such that

$$
\tilde{A} V_{i}=V_{i} \tilde{A}, \quad i=1, \ldots, n .
$$

Indeed, since $A \in C^{*}\left(T_{1}, \ldots, T_{n}\right)^{\prime}$, it is easy to see that

$$
\left(\oplus_{j=1}^{n} A\right) D_{\mathcal{T}}(\underbrace{0, \ldots, 0}_{i-1 \text { times }}, h, 0, \ldots, 0)=D_{\mathcal{T}}(\underbrace{0, \ldots, 0}_{i-1 \text { times }}, A h, 0, \ldots, 0)
$$

for any $h \in \mathcal{H}$ and $i=1, \ldots, n$. Since $\mathcal{K}=\bigvee_{\alpha \in \mathbb{F}_{n}^{+}} V_{\alpha} \mathcal{H}$, one can see that $\tilde{A}$ is uniquely determined by condition (2.3), and, moreover,

$$
\tilde{A}\left(\sum_{\alpha \in \mathbb{F}_{n}^{+}} e_{\alpha} \otimes d_{(\alpha)}\right)=\sum_{\alpha \in \mathbb{F}_{n}^{+}} e_{\alpha} \otimes\left(\oplus_{j=1}^{n} A\right) d_{(\alpha)}
$$

for any

$$
\sum_{\alpha \in \mathbb{F}_{n}^{+}} e_{\alpha} \otimes d_{(\alpha)} \in F^{2}\left(H_{n}\right) \otimes \mathcal{D}
$$

It is easy to see that the mapping $A \mapsto \tilde{A}$ from $C^{*}\left(T_{1}, \ldots, T_{n}\right)^{\prime}$ to $C^{*}\left(V_{1}, \ldots, V_{n}\right)^{\prime}$ is a *-monomorphism of $C^{*}$-algebras. Notice also that if we set $A_{k}:=\left.\tilde{A}\right|_{\mathcal{H}_{k}}, k=1,2, \ldots$, then $A_{k}$ is the unique extension of $A_{k-1}$ to $\mathcal{H}_{k}$ such that $A_{k} \in C^{*}\left(V_{1, k}, \ldots, V_{n, k}\right)^{\prime}$. 
Let $\mathcal{H}_{1}, \mathcal{H}_{2}, \mathcal{K}_{1}, \mathcal{K}_{2}$ be Hilbert spaces and denote $\mathcal{H}:=\mathcal{H}_{1} \oplus \mathcal{H}_{2}$ and $\mathcal{K}=: \mathcal{K}_{1} \oplus \mathcal{K}_{2}$. Any operator $T \in B(\mathcal{H}, \mathcal{K})$ can be written as an operator matrix

$$
T=\left[\begin{array}{ll}
A & B \\
C & X
\end{array}\right]
$$

with respect to the corresponding decompositions of $\mathcal{H}$ and $\mathcal{K}$. It was proved in [12] that

$$
\inf _{X \in B\left(\mathcal{H}_{2}, \mathcal{K}_{2}\right)}\left\|\left[\begin{array}{ll}
A & B \\
C & X
\end{array}\right]\right\|=\max \left\{\left\|\left[\begin{array}{l}
A \\
C
\end{array}\right]\right\|,\left\|\left[\begin{array}{ll}
A & B
\end{array}\right]\right\|\right\} .
$$

Moreover, there are sequences of real numbers $\left\{c_{k}\right\},\left\{d_{k}\right\}$ such that

$$
Y:=\text { WOT- } \lim _{k \rightarrow \infty}-c_{k} C\left(I-d_{k} A^{*} A\right)^{-1} A^{*} B
$$

exists and the above-mentioned infimum is equal to

$$
\left\|\left[\begin{array}{ll}
A & B \\
C & Y
\end{array}\right]\right\|
$$

In what follows, we find a non-commutative multivariable analogue of Parrott's generalization $[\mathbf{1 2}]$ of the Sz.-Nagy-Foiaş commutant lifting theorem [24].

Theorem 2.1. Let $\mathcal{T}:=\left[T_{1}, \ldots, T_{n}\right]$ be a contractive sequence of operators on a Hilbert space $\mathcal{H}$ and let $\mathcal{V}:=\left[V_{1}, \ldots, V_{n}\right]$ be its minimal isometric dilation on the Hilbert space $\mathcal{K} \supseteq \mathcal{H}$. If $X \in B(\mathcal{H})$ and $X T_{i}=T_{i} X$ for any $i=1, \ldots, n$, then there exists $X_{\infty} \in B(\mathcal{K})$ satisfying the following properties:

(i) $X_{\infty} V_{i}=V_{i} X_{\infty}$, for any $i=1, \ldots, n$;

(ii) $X_{\infty} \tilde{A}=\tilde{A} X_{\infty}$ for any $A \in C^{*}\left(T_{1}, \ldots T_{n}, X\right)^{\prime}$;

(iii) $\left.X_{\infty}^{*}\right|_{\mathcal{H}}=X^{*}$ and $\left.P_{\mathcal{H}} \tilde{A} V_{\alpha} X_{\infty}^{* m} V_{\beta}^{*}\right|_{\mathcal{H}}=A T_{\alpha} X^{* m} T_{\beta}^{*}$, for any $\alpha, \beta \in \mathbb{F}_{n}^{+}, A \in$ $C^{*}\left(T_{1}, \ldots T_{n}, X\right)^{\prime}$, and $m \in \mathbb{N}$;

(iv) $\left\|X_{\infty}\right\|=\|X\|$.

Proof. We use the notation and preliminaries preceding the theorem. Let us construct a sequence of operators $X_{k} \in B\left(\mathcal{H}_{k}\right), k=1,2, \ldots$, with the following properties:

(a) $X_{k} V_{i, k}=V_{i, k} X_{k}$, for any $i=1, \ldots, n$;

(b) $X_{k} A_{k}=A_{k} X_{k}$ for any $A \in C^{*}\left(T_{1}, \ldots T_{n}, X\right)^{\prime}$;

(c) $\left.X_{k}^{*}\right|_{\mathcal{H}_{k-1}}=X_{k-1}^{*}$ and $\left.P_{\mathcal{H}_{k-1}} A_{k} V_{\alpha, k} X_{k}^{* m} V_{\beta, k}^{*}\right|_{\mathcal{H}_{k-1}}=A_{k-1} V_{\alpha, k-1} X_{k-1}^{* m} V_{\beta, k-1}^{*}$, for any $A \in C^{*}\left(T_{1}, \ldots T_{n}, X\right)^{\prime}$ and $\alpha, \beta \in \mathbb{F}_{n}^{+}, m \in \mathbb{N}$;

(d) $\left\|X_{k}\right\|=\left\|X_{k-1}\right\|=\|X\|$. 
Once this is established, it will be a routine exercise to show that the limit $X_{\infty}:=$ SOT- $\lim _{k \rightarrow \infty} X_{k} P_{\mathcal{H}_{k}}$ exists and $X_{\infty}$ has the stated properties in the theorem.

Our first step is to show that $X_{1}$ exists with the above-mentioned properties, when $k=1$. For each $i=1,2, \ldots, n$, let $V_{i, 1}: \mathcal{H}_{1}:=\mathcal{H} \oplus \mathcal{D} \rightarrow \mathcal{H}_{1}$ be defined by

$$
V_{i, 1}(h \oplus d)=T_{i} h \oplus D(\underbrace{0, \ldots, 0}_{i-1 \text { times }}, h, 0, \ldots, 0) .
$$

Notice that $V_{i, 1}, i=1,2, \ldots, n$, are partial isometries with orthogonal ranges and initial space $\mathcal{H} \oplus\{0\}$. Define $Z \in B\left(\mathcal{H}_{1}\right)$ by $Z(h \oplus d):=X h \oplus 0$. Let

$$
\mathcal{N}_{1}:=\mathcal{H} \oplus\{0\}, \quad \mathcal{N}_{2}:=\mathcal{H}_{1} \ominus \mathcal{N}_{1}=\{0\} \oplus \mathcal{D}, \quad \mathcal{M}_{1}:=V_{1,1} \mathcal{N}_{1}+\cdots+V_{n, 1} \mathcal{N}_{1},
$$

and $\mathcal{M}_{2}:=\mathcal{H}_{1} \ominus \mathcal{M}_{1}$.

Now we shall prove that the set of all operators $X_{1} \in B\left(\mathcal{H}_{1}\right)$ satisfying the relations $\left.P_{\mathcal{H}} X_{1}\right|_{\mathcal{H}}=X$ and $X_{1} V_{i, 1}=V_{i, 1} X_{1}$, for any $i=1, \ldots, n$, is non-empty and is exactly $L_{1}+B\left(\mathcal{M}_{2}, \mathcal{N}_{2}\right)$ where $B\left(\mathcal{M}_{2}, \mathcal{N}_{2}\right)$ is embedded in $B\left(\mathcal{H}_{1}\right)$, and $L_{1} \in B\left(\mathcal{H}_{1}\right)$ is defined by

$$
L_{1}:=\sum_{i=1}^{n} V_{i, 1} X V_{i, 1}^{*}+Z P_{\mathcal{M}_{2}}
$$

Since $\sum_{i=1}^{n} V_{i, 1} V_{i, 1}^{*}=P_{\mathcal{M}_{1}}, V_{i, 1}^{*} V_{i, 1}=P_{\mathcal{N}_{1}}$ for any $i=1,2, \ldots, n$, and $V_{i, 1}^{*} V_{j, 1}=0$ if $i \neq j$, we infer that

$$
L_{1} P_{\mathcal{M}_{1}}=\left(\sum_{i=1}^{n} V_{i, 1} X V_{i, 1}^{*}\right) P_{\mathcal{M}_{1}}=\left(\sum_{i=1}^{n} V_{i, 1} X V_{i, 1}^{*}\right)\left(\sum_{j=1}^{n} V_{j, 1} V_{j, 1}^{*}\right)=\sum_{i=1}^{n} V_{i, 1} X V_{i, 1}^{*} .
$$

Therefore,

$$
L_{1} P_{\mathcal{M}_{1}}=\sum_{i=1}^{n} V_{i, 1} X V_{i, 1}^{*}
$$

On the other hand, we have

$$
\begin{aligned}
P_{\mathcal{N}_{1}} L_{1} & =P_{\mathcal{N}_{1}} L_{1} P_{\mathcal{M}_{1}}+P_{\mathcal{N}_{1}} L_{1} P_{\mathcal{M}_{2}} \\
& =P_{\mathcal{N}_{1}}\left(\sum_{i=1}^{n} V_{i, 1} X V_{i, 1}^{*}\right)+P_{\mathcal{N}_{1}}\left(\sum_{i=1}^{n} V_{i, 1} X V_{i, 1}^{*}+Z P_{\mathcal{M}_{2}}\right) P_{\mathcal{M}_{2}} \\
& =\sum_{i=1}^{n} T_{i} X V_{i, 1}^{*}+P_{\mathcal{N}_{1}} Z P_{\mathcal{M}_{2}}=\sum_{i=1}^{n} X T_{i} V_{i, 1}^{*}+P_{\mathcal{N}_{1}} Z P_{\mathcal{M}_{2}} \\
& =X P_{\mathcal{N}_{1}} P_{\mathcal{M}_{1}}+P_{\mathcal{N}_{1}} Z P_{\mathcal{M}_{2}}=P_{\mathcal{N}_{1}} Z\left(P_{\mathcal{M}_{1}}+P_{\mathcal{M}_{2}}\right)=P_{\mathcal{N}_{1}} Z=Z .
\end{aligned}
$$

Therefore,

$$
P_{\mathcal{N}_{1}} L_{1}=Z
$$


which implies that $\left.P_{\mathcal{N}_{1}} L_{1}\right|_{\mathcal{N}_{1}}=X$. Using the above-mentioned relations and $V_{i} P_{\mathcal{N}_{1}}=V_{i}$, $i=1, \ldots, n$, we deduce

$$
\begin{aligned}
L_{1} V_{i, 1} & =L_{1} P_{\mathcal{M}_{1}} V_{i, 1}=\left(\sum_{j=1}^{n} V_{j, 1} X V_{j, 1}^{*}\right) V_{i, 1} \\
& =V_{i, 1} X V_{i, 1}^{*} V_{i, 1}=V_{i, 1} X P_{\mathcal{N}_{1}}=V_{i, 1} Z \\
& =V_{i, 1} P_{\mathcal{N}_{1}} L_{1} .
\end{aligned}
$$

Therefore,

$$
L_{1} V_{i, 1}=V_{i, 1} L_{1}, \quad \text { for any } i=1, \ldots, n .
$$

Now, suppose that $X_{1} \in B\left(\mathcal{H}_{1}\right)$ satisfies $\left.P_{\mathcal{H}} X_{1}\right|_{\mathcal{H}}=X$ and $X_{1} V_{i, 1}=V_{i, 1} X_{1}$ for any $i=1, \ldots, n$. Since $X_{1}-L_{1}$ commutes with each $V_{i, 1}, i=1, \ldots, n$, and $\mathcal{N}_{2}=\operatorname{ker} V_{i, 1}$, we infer that $\mathcal{N}_{2}$ is invariant for $X_{1}-L_{1}$. Taking into account that $\left.P_{\mathcal{N}_{1}}\left(X_{1}-L_{1}\right)\right|_{\mathcal{N}_{1}}=0$, we deduce that the range of $X_{1}-L_{1}$ is in $\mathcal{N}_{2}$.

On the other hand, using $V_{i, 1} P_{\mathcal{N}_{1}}=V_{i, 1}$, we obtain

$$
\begin{aligned}
\left(X_{1}-L_{1}\right) P_{\mathcal{M}_{1}} & =\left(X_{1}-L_{1}\right) \sum_{i=1}^{n} V_{i, 1} V_{i, 1}^{*}=\sum_{i=1}^{n} V_{i, 1}\left(X_{1}-L_{1}\right) V_{i, 1}^{*} \\
& =\left.\sum_{i=1}^{n} V_{i, 1} P_{\mathcal{N}_{1}}\left(X_{1}-L_{1}\right)\right|_{\mathcal{N}_{1}} V_{i, 1}^{*}=0
\end{aligned}
$$

This shows that $X_{1}-L_{1} \in B\left(\mathcal{M}_{2}, \mathcal{H}_{1}\right)$. Summing up, we deduce that $X_{1}-L_{1} \in$ $B\left(\mathcal{M}_{2}, \mathcal{N}_{2}\right)$, which shows that $X_{1}=L_{1}+Y$ for some $Y \in B\left(\mathcal{M}_{2}, \mathcal{N}_{2}\right)$.

Conversely, let $Y \in B\left(\mathcal{M}_{2}, \mathcal{N}_{2}\right)$. It is clear that $\left.P_{\mathcal{N}_{1}}\left(L_{1}+Y\right)\right|_{\mathcal{N}_{1}}=X$, and, since $V_{i, 1} Y=Y V_{i, 1}=0, i=1, \ldots, n$, we infer that $L_{1}+Y$ commutes with each $V_{i, 1}, i=$ $1, \ldots, n$. Therefore, we proved that the set

$$
\left\{X_{1} \in B\left(\mathcal{H}_{1}\right):\left.P_{\mathcal{H}} X_{1}\right|_{\mathcal{H}}=X \text { and } V_{i, 1} X_{1}=X_{1} V_{i, 1}\right\}
$$

is non-empty and equal to $L_{1}+B\left(\mathcal{M}_{2}, \mathcal{N}_{2}\right)$, where $L_{1}$ is given by (2.5). According to (2.4), there exists $X_{1}$ with

$$
\left\|X_{1}\right\|=\max \left\{\left\|L_{1} P_{\mathcal{M}_{1}}\right\|,\left\|P_{\mathcal{N}_{1}} L_{1}\right\|\right\} .
$$

Taking into account (2.6), (2.7), we obtain $\left\|X_{1}\right\|=\|X\|$. Moreover, if we set

$$
\begin{aligned}
\Lambda & :=P_{\mathcal{N}_{1}} L_{1} P_{\mathcal{M}_{1}}=Z P_{\mathcal{M}_{1}}, \\
\Delta & :=P_{\mathcal{N}_{2}} L_{1} P_{\mathcal{M}_{1}}=P_{\mathcal{N}_{2}}\left(\sum_{i=1}^{n} V_{i, 1} Z V_{i, 1}^{*}\right), \\
\Gamma & :=P_{\mathcal{N}_{1}} L_{1} P_{\mathcal{M}_{2}}=Z P_{\mathcal{M}_{2}}
\end{aligned}
$$


then we may choose $X_{1}$ to be of the form

$$
X_{1}=\Lambda+\Delta+\Gamma+\text { WOT- } \lim _{k \rightarrow \infty}-c_{k} \Delta\left(I-d_{k} \Lambda \Lambda^{*}\right)^{-1} \Lambda^{*} \Gamma
$$

for some sequences of real numbers $\left\{c_{k}\right\},\left\{d_{k}\right\}$.

Now, let $A \in C^{*}\left(T_{1}, \ldots, T_{n}, X\right)^{\prime}$ and $A_{1}$ be its canonical extension to $\mathcal{H}_{1}$. Since $A_{1} \in$ $C^{*}\left(V_{1,1}, \ldots, V_{n, 1}\right)^{\prime}$ and $P_{\mathcal{M}_{1}}=\sum_{i=1}^{n} V_{i, 1} V_{i, 1}^{*}$, it is clear that $\mathcal{M}_{1}$ and $\mathcal{M}_{2}$ are invariant under $A_{1}$. It follows from the definition of $A_{1}$ that $\mathcal{N}_{1}$ and $\mathcal{N}_{2}$ are also invariant to $A_{1}$. Since $A_{1} \in C^{*}\left(V_{1,1}, \ldots, V_{n, 1}, Z\right)^{\prime}$, it follows that $A_{1}$ commutes with $\Lambda, \Delta, \Gamma$, and, hence, with $X_{1}$. Let us show that

$$
\left.P_{\mathcal{H}} A_{1} V_{\alpha, 1} X_{1}^{* m} V_{\beta, 1}^{*}\right|_{\mathcal{H}}=A T_{\alpha} X^{* m} T_{\beta}^{*},
$$

for any $A \in C^{*}\left(T_{1}, \ldots T_{n}, X\right)^{\prime}$ and $\alpha, \beta \in \mathbb{F}_{n}^{+}, m \in \mathbb{N}$. Since $\mathcal{N}_{2}$ is invariant for $X_{1}$ and $P_{\mathcal{H}} X_{1}=Z$, we have

$$
X_{1}=\left[\begin{array}{ll}
X & 0 \\
* & *
\end{array}\right]
$$

with respect to the orthogonal decomposition $\mathcal{H}_{1}=\mathcal{H} \oplus \mathcal{H}^{\perp}$, where $\mathcal{H}^{\perp}:=\mathcal{H}_{1} \ominus \mathcal{H}$. Notice that $\left.X_{1}^{*}\right|_{\mathcal{H}}=X^{*}$. On the other hand, the matrices of $V_{i, 1}$ and $A_{1}$ are of the form

$$
V_{i, 1}=\left[\begin{array}{cc}
T_{i} & 0 \\
* & 0
\end{array}\right] \quad \text { and } \quad A_{1}=\left[\begin{array}{cc}
A & 0 \\
0 & *
\end{array}\right]
$$

Hence,

$$
\left.A_{1} V_{\alpha, 1} X_{1}^{m} V_{\beta, 1}^{*}\right|_{\mathcal{H}}=\left[\begin{array}{cc}
A T_{\alpha} X^{m} T_{\beta}^{*} & 0 \\
* & 0
\end{array}\right],
$$

which proves (2.8).

Now, since $X_{1}$ always exists, one can replace $T_{1}, \ldots, T_{n}$ and $X$ by $V_{1,1}, \ldots, V_{n, 1}$ and $X_{1}$ and iterate the process, obtaining at the $k$ th step an operator $X_{k} \in B\left(\mathcal{H}_{k}\right)$ satisfying properties (a)-(d). The proof is complete.

As in the classical case, we expect Theorem 2.1 to yield interpolation theorems for analytic functions from the open unit ball of $\mathbb{C}^{n}$ into a von Neumann algebra. We need to consider some preliminary results. According to Theorem 1.2 from [19], the commutant of $F_{n}^{\infty}$, which we denote by $R_{n}^{\infty}$, is equal to $U^{*} F_{n}^{\infty} U$, where $U$ is the unitary operator on $F^{2}\left(H_{n}\right)$ defined by $U\left(e_{i_{1}} \otimes e_{i_{2}} \otimes \cdots \otimes e_{i_{k}}\right)=e_{i_{k}} \otimes \cdots \otimes e_{i_{2}} \otimes e_{i_{1}}$. Moreover, the commutant of $R_{n}^{\infty}$ is equal to $F_{n}^{\infty}$.

For each $\mathcal{S} \subseteq B(\mathcal{H})$, we denote its commutant by $\mathcal{S}^{\prime}$. Define the isometries $Q_{\alpha}: \mathcal{H} \rightarrow$ $F^{2}\left(H_{n}\right) \otimes \mathcal{H}, \alpha \in \mathbb{F}_{n}^{+}$, by $Q_{\alpha} h:=e_{\alpha} \otimes h, h \in \mathcal{H}$.

Lemma 2.2. If $\mathcal{S} \subseteq B(\mathcal{H})$ and $f$ is in the commutant of

$$
\left\{U^{*} S_{i} U \otimes I_{\mathcal{H}}: i=1, \ldots, n\right\} \cup\left\{I_{F^{2}\left(H_{n}\right)} \otimes Y: Y \in \mathcal{S}\right\},
$$


then the operators $Q_{\alpha}^{*} f Q_{e}, \alpha \in \mathbb{F}_{n}^{+}$, are in $\mathcal{S}^{\prime}$, and $f$ has a formal Fourier expansion

$$
f \sim \sum_{\alpha \in \mathbb{F}_{n}^{+}} S_{\alpha} \otimes Q_{\alpha}^{*} f Q_{e}
$$

Proof. Since $f$ commutes with $U^{*} S_{i} U \otimes I_{\mathcal{H}}, i=1, \ldots, n$, we infer that it is uniquely determined by

$$
f(1 \otimes h)=\sum_{\alpha \in \mathbb{F}_{n}^{+}} e_{\alpha} \otimes Q_{\alpha}^{*} f Q_{e} h, \quad h \in \mathcal{H}
$$

Indeed, notice that

$$
\begin{aligned}
f\left(e_{\beta} \otimes h\right) & =f\left(U^{*} S_{\beta} U \otimes I_{\mathcal{H}}\right)(1 \otimes h)=\left(U^{*} S_{\beta} U \otimes I_{\mathcal{H}}\right) f(1 \otimes h) \\
& =\sum_{\alpha \in \mathbb{F}_{n}^{+}} e_{\alpha \beta} \otimes Q_{\alpha}^{*} f Q_{e} h .
\end{aligned}
$$

On the other hand, since $f$ commutes with each $I_{F^{2}\left(H_{n}\right)} \otimes Y, Y \in \mathcal{S}$, we have

$$
\begin{aligned}
\left(I_{F^{2}\left(H_{n}\right)} \otimes Y\right) f(1 \otimes h) & =\sum_{\alpha \in \mathbb{F}_{n}^{+}} e_{\alpha} \otimes Y Q_{\alpha}^{*} f Q_{e} h \\
& =f\left(I_{F^{2}\left(H_{n}\right)} \otimes Y\right)(1 \otimes h)=\sum_{\alpha \in \mathbb{F}_{n}^{+}} e_{\alpha} \otimes Q_{\alpha}^{*} f Q_{e} Y h .
\end{aligned}
$$

Hence, $Q_{\alpha}^{*} f Q_{e} \in \mathcal{S}^{\prime}$.

We denote by $F_{n}^{\infty} \otimes \mathcal{S}$ (respectively, $R_{n}^{\infty} \otimes \mathcal{S}$ ) the spatial tensor product, and by $F_{n}^{\infty} \bar{\otimes} \mathcal{S}^{\prime}$ (respectively, $R_{n}^{\infty} \bar{\otimes} \mathcal{S}^{\prime}$ ) the WOT-closed algebra generated by the spatial tensor product of the two algebras. The following result is a Tomita-type theorem in a non-self-adjoint setting.

Theorem 2.3. If $\mathcal{S} \subseteq B(\mathcal{H})$ and $I_{\mathcal{H}} \in \mathcal{S}$, then

$$
\left(R_{n}^{\infty} \otimes \mathcal{S}\right)^{\prime}=F_{n}^{\infty} \bar{\otimes} \mathcal{S}^{\prime} \quad \text { and } \quad\left(F_{n}^{\infty} \otimes \mathcal{S}\right)^{\prime}=R_{n}^{\infty} \bar{\otimes} \mathcal{S}^{\prime} .
$$

Proof. Since $\left(R_{n}^{\infty}\right)^{\prime}=F_{n}^{\infty}$, it is easy to see that $F_{n}^{\infty} \bar{\otimes} \mathcal{S}^{\prime} \subseteq\left(R_{n}^{\infty} \otimes \mathcal{S}\right)^{\prime}$. Conversely, assume that $f$ is in $\left(R_{n}^{\infty} \otimes \mathcal{S}\right)^{\prime}$. Then $f$ belongs to the commutant of the set $\left\{U^{*} S_{i} U \otimes I_{\mathcal{H}}\right.$ : $i=1, \ldots, n\} \cup\{I \otimes Y: Y \in \mathcal{S}\}$. According to Lemma 2.2 , the operators $W_{(\alpha)}:=Q_{\alpha}^{*} f Q_{e}$, $\alpha \in \mathbb{F}_{n}^{+}$, are in $\mathcal{S}^{\prime}$, and $f$ has a formal Fourier expansion

$$
f \sim \sum_{\alpha \in \mathbb{F}_{n}^{+}} S_{\alpha} \otimes W_{(\alpha)}
$$

For each $0<r<1$, define

$$
f_{r}:=\sum_{\alpha \in \mathbb{F}_{n}^{+}} r^{|\alpha|} S_{\alpha} \otimes W_{(\alpha)} .
$$

Notice that the convergence of this series is in the uniform norm. Indeed, since

$$
f(1 \otimes h)=\sum_{\alpha \in \mathbb{F}_{n}^{+}} e_{\alpha} \otimes W_{(\alpha)} h, \quad h \in \mathcal{H}
$$


we infer that

$$
\sum_{\alpha \in \mathbb{F}_{n}^{+}} W_{(\alpha)}^{*} W_{(\alpha)} \leqslant\|f\| I_{\mathcal{H}}
$$

On the other hand, we have

$$
\begin{aligned}
\sum_{k=0}^{\infty} r^{k}\left\|\sum_{|\alpha|=k} S_{\alpha} \otimes W_{(\alpha)}\right\| & =\sum_{k=0}^{\infty} r^{k}\left\|\sum_{|\alpha|=k} W_{(\alpha)}^{*} W_{(\alpha)}\right\|^{1 / 2} \\
& \leqslant\left(\sum_{k=0}^{\infty} r^{k}\right)\left\|\sum_{\alpha \in \mathbb{F}_{n}^{+}} W_{(\alpha)}^{*} W_{(\alpha)}\right\|^{1 / 2} .
\end{aligned}
$$

Therefore, $f_{r} \in F_{n}^{\infty} \bar{\otimes} \mathcal{S}^{\prime}$. Moreover, $f_{r}$ is in the norm-closure of the algebra generated by the operators $S_{i} \otimes I, I \otimes Z$, where $Z \in \mathcal{S}^{\prime}, i=1, \ldots, n$. According to the non-commutative von Neumann inequality, we have $\left\|f_{r}\right\| \leqslant\|f\|$ for any $0<r<1$. Now, let us prove that

$$
\text { SOT-lim } \lim _{r \rightarrow 1}=f \text {. }
$$

For any $h \in \mathcal{H}, \beta \in \mathbb{F}_{n}^{+}$, we have

$$
\begin{aligned}
\left\|f_{r}\left(e_{\beta} \otimes h\right)-f\left(e_{\beta} \otimes h\right)\right\|^{2} & =\left\|\sum_{\alpha \in \mathbb{F}_{n}^{+}}\left(r^{|\alpha|}-1\right) e_{\alpha \beta} \otimes W_{(\alpha)} h\right\|^{2} \\
& =\sum_{\alpha \in \mathbb{F}_{n}^{+}}\left(r^{|\alpha|}-1\right)^{2}\left\|W_{(\alpha)} h\right\|^{2} \\
& =\sum_{k=1}^{\infty}\left(r^{k}-1\right)^{2} \sum_{|\alpha|=k}\left\|W_{(\alpha)} h\right\|^{2} .
\end{aligned}
$$

Using (2.9), we deduce that $\left\|f_{r}\left(e_{\beta} \otimes h\right)-f\left(e_{\beta} \otimes h\right)\right\| \rightarrow 0$, as $r \rightarrow 1$. Since $\left\|f_{r}\right\| \leqslant\|f\|$, a standard argument shows that (2.10) follows. Hence, $f \in F_{n}^{\infty} \bar{\otimes} \mathcal{S}^{\prime}$ and the proof is complete.

One can easily see from the proof of Theorem 2.3 that $F_{n}^{\infty} \bar{\otimes} \mathcal{S}^{\prime}$ is the WOT-closed algebra generated by the operators $S_{i} \otimes I, I \otimes Z$, where $i=1, \ldots, n$, and $Z \in \mathcal{S}^{\prime}$, and any $f \in F_{n}^{\infty} \bar{\otimes} \mathcal{S}^{\prime}$ has a formal Fourier expansion. On the other hand, if $\mathcal{M}$ is a von Neumann algebra, then $\left(F_{n}^{\infty} \bar{\otimes} \mathcal{M}\right)^{\prime \prime}=F_{n}^{\infty} \bar{\otimes} \mathcal{M}$.

A complete description of the invariant subspace structure of $F_{n}^{\infty}$ was obtained in [15, Theorem 2.2] (even in a more general setting). A subspace $\mathcal{N}$ of $F^{2}\left(H_{n}\right)$ is invariant under $S_{1}, \ldots, S_{n}$ if and only if $\mathcal{N}=\bigoplus_{\lambda \in \Lambda} U^{*} \varphi_{\lambda} U\left[F^{2}\left(H_{n}\right)\right]$, for some family $\left\{\varphi_{\lambda} \in F_{n}^{\infty}: \lambda \in \Lambda\right\}$ of isometries with orthogonal ranges (see also $[\mathbf{8}, \mathbf{1 9}]$ ).

In what follows we use Theorems 2.1 and 2.3 in order to extend Sarason's interpolation result $[\mathbf{2 3}]$ to our setting.

Theorem 2.4. Let $\mathcal{M} \subseteq B(\mathcal{K})$ be such that $I_{\mathcal{H}} \in \mathcal{M}=\mathcal{M}^{*}$ and let $\mathcal{N} \subseteq F^{2}\left(H_{n}\right)$ be an invariant subspace under $S_{1}^{*}, \ldots, S_{n}^{*}$. If $X \in B(\mathcal{N} \otimes \mathcal{K})$ commutes with $P_{\mathcal{N} \otimes \mathcal{K}}\left(F_{n}^{\infty} \otimes\right.$ 
$\mathcal{M})\left.\right|_{\mathcal{N} \otimes \mathcal{K}}$, then there exists $\Phi \in R_{n}^{\infty} \bar{\otimes} \mathcal{M}^{\prime}$ such that

$$
\left.P_{\mathcal{N} \otimes \mathcal{K}} \Phi\right|_{\mathcal{N} \otimes \mathcal{K}}=X, \quad\|\Phi\|=\|X\|,
$$

where $P_{\mathcal{N} \otimes \mathcal{K}}$ is the orthogonal projection of $F^{2}\left(H_{n}\right) \otimes \mathcal{K}$ onto $\mathcal{N} \otimes \mathcal{K}$. If, in addition, $U \mathcal{N}$ is an invariant subspace under $S_{1}^{*}, \ldots, S_{n}^{*}$, then

$$
\left[\left.P_{\mathcal{N} \otimes \mathcal{K}}\left(F_{n}^{\infty} \otimes \mathcal{M}\right)\right|_{\mathcal{N} \otimes \mathcal{K}}\right]^{\prime}=\left.P_{\mathcal{N} \otimes \mathcal{K}}\left[\left(F_{n}^{\infty}\right)^{\prime} \bar{\otimes} \mathcal{M}^{\prime}\right]\right|_{\mathcal{N} \otimes \mathcal{K}}
$$

Proof. Let $B \in \mathcal{M}$, and define $\tilde{B} \in B\left(F^{2}\left(H_{n}\right) \otimes \mathcal{K}\right)$ by $\tilde{B}:=I \otimes B$. Since $X$ commutes with $\left.P_{\mathcal{N} \otimes \mathcal{K}}\left(F_{n}^{\infty} \otimes \mathcal{M}\right)\right|_{\mathcal{N} \otimes \mathcal{K}}$ and $I_{\mathcal{H}} \in \mathcal{M}$, we deduce that $X$ commutes with $T_{i}:=\left.P_{\mathcal{N}} S_{i}\right|_{\mathcal{N}} \otimes I_{\mathcal{K}}, i=1, \ldots, n$, and also with $\left.\tilde{B}\right|_{\mathcal{N} \otimes \mathcal{K}}=I_{\mathcal{N}} \otimes B$ for any $B \in \mathcal{M}$. Since $\mathcal{M}=\mathcal{M}^{*}$, we have $\left.\tilde{B}\right|_{\mathcal{N} \otimes \mathcal{K}} \in C^{*}\left(T_{1}, \ldots, T_{n}, X\right)^{\prime}$. On the other hand, $\mathcal{N}$ is invariant under each $S_{1}^{*}, \ldots, S_{n}^{*}$, and $\left[\left.P_{\mathcal{N}} S_{1}\right|_{\mathcal{N}}, \ldots,\left.P_{\mathcal{N}} S_{n}\right|_{\mathcal{N}}\right]$ is a $C_{0}$-row contraction. Using [14, Proposition 2.3] we infer that its minimal isometric dilation is $\left[S_{1}, \ldots, S_{n}\right]$. Therefore, the minimal isometric dilation of $\left[T_{1}, \ldots, T_{n}\right]$ is $\left[S_{1} \otimes I_{\mathcal{K}}, \ldots, S_{n} \otimes I_{\mathcal{K}}\right]$. On the other hand, $\tilde{B}$ commutes with $\left\{S_{i} \otimes I_{\mathcal{K}}, S_{i}^{*} \otimes I_{\mathcal{K}}, i=1, \ldots, n\right\}$. Notice that $\tilde{B}$ must be the canonical extension of $\left.\tilde{B}\right|_{\mathcal{N} \otimes \mathcal{K}}$. According to Theorem 2.1, there exists $X_{\infty} \in B\left(F^{2}\left(H_{n}\right) \otimes \mathcal{K}\right)$ such that

(i) $X_{\infty}\left(S_{i} \otimes I_{\mathcal{K}}\right)=\left(S_{i} \otimes I_{\mathcal{K}}\right) X_{\infty}$, for any $i=1, \ldots, n$;

(ii) $X_{\infty} \tilde{B}=\tilde{B} X_{\infty}$ for any $B \in \mathcal{M}$;

(iii) $\left\|X_{\infty}\right\|=\|X\|$;

(iv) $\left.X_{\infty}^{*}\right|_{\mathcal{N} \otimes \mathcal{K}}=X^{*}$.

Using Theorem 2.3, we find $\Phi$ in $R_{n}^{\infty} \bar{\otimes} \mathcal{M}^{\prime}$ such that $X_{\infty}=\Phi$. Now, assume that $U \mathcal{N}$ is an invariant subspace under $S_{1}^{*}, \ldots, S_{n}^{*}$, and let $X:=\left.P_{\mathcal{N} \otimes \mathcal{K}} \Psi\right|_{\mathcal{N} \otimes \mathcal{K}}$ with $\Psi \in R_{n}^{\infty} \bar{\otimes} \mathcal{M}^{\prime}$. Notice that $X$ commutes with $\left.P_{\mathcal{N} \otimes \mathcal{K}}\left(F_{n}^{\infty} \otimes \mathcal{M}\right)\right|_{\mathcal{N} \otimes \mathcal{K}}$. The proof is complete.

Corollary 2.5. Let $\mathcal{M}$ be a von Neumann algebra acting on a Hilbert space $\mathcal{K}$ and let $\mathcal{N} \subseteq F^{2}\left(H_{n}\right)$ be a subspace with the property that $\mathcal{N}$ and $U \mathcal{N}$ are invariant under $S_{1}^{*}, \ldots, S_{n}^{*}$. If $\Psi \in R_{n}^{\infty} \bar{\otimes} \mathcal{M}$, then there is $\Phi \in R_{n}^{\infty} \bar{\otimes} \mathcal{M}$ such that $\|\Phi\|=\left\|\left.P_{\mathcal{N} \otimes \mathcal{K}} \Psi\right|_{\mathcal{N} \otimes \mathcal{K}}\right\|$ and $\left.P_{\mathcal{N} \otimes \mathcal{K}} \Phi\right|_{\mathcal{N} \otimes \mathcal{K}}=\left.P_{\mathcal{N} \otimes \mathcal{K}} \Psi\right|_{\mathcal{N} \otimes \mathcal{K}}$.

Proof. Since $X:=\left.P_{\mathcal{N} \otimes \mathcal{K}} \Psi\right|_{\mathcal{N} \otimes \mathcal{K}}$ commutes with $\left.P_{\mathcal{N} \otimes \mathcal{K}}\left(F_{n}^{\infty} \bar{\otimes} \mathcal{M}^{\prime}\right)\right|_{\mathcal{N} \otimes \mathcal{K}}$, we can use Theorem 2.4 to find $\Phi \in R_{n}^{\infty} \bar{\otimes}\left(\mathcal{M}^{\prime}\right)^{\prime}$ with the required properties. Since $\left(\mathcal{M}^{\prime}\right)^{\prime}=\mathcal{M}$, according to the double commutant theorem, the result follows.

Let $J$ be a WOT-closed two-sided ideal of $F_{n}^{\infty}$. Define $J(1):=\{\psi(1) ; \psi \in J\}$ and $\mathcal{N}_{J}:=F^{2}\left(H_{n}\right) \ominus J(1)$. Let us remark that $\mathcal{N}_{J}$ and $U \mathcal{N}_{J}$ are invariant subspaces under $S_{i}^{*}, i=1, \ldots, n$. It was proved in $[\mathbf{3}, \mathbf{7}]$ that the quotient algebra $F_{n}^{\infty} / J$ is completely isometricaly isomorphic to $\left.P_{\mathcal{N}_{J}} F_{n}^{\infty}\right|_{\mathcal{N}_{J}}$. Using this result and Theorem 2.4, one can deduce the following Tomita-type commutant result. 
Corollary 2.6. If $J$ is a WOT-closed two-sided ideal in $F_{n}^{\infty}$ and $\mathcal{M} \subseteq B(\mathcal{K})$ such that $I_{\mathcal{H}} \in \mathcal{M}=\mathcal{M}^{*}$, then

$$
\left[\left(F_{n}^{\infty} / J\right) \otimes \mathcal{M}\right]^{\prime}=\left(F_{n}^{\infty} / J\right)^{\prime} \bar{\otimes}(\mathcal{M})^{\prime} .
$$

In the particular case when $J$ is the WOT-closed two-sided ideal in $F_{n}^{\infty}$ generated by $\left\{e_{j} \otimes e_{i}-e_{i} \otimes e_{j}: 1 \leqslant i<j \leqslant n\right\}$, we have $\mathcal{N}_{J}=F_{s}^{2}$, the symmetric Fock space, and $F_{n}^{\infty} / J$ can be identified with $\mathcal{W}_{n}^{\infty}:=\left.P_{F_{s}^{2}} F_{n}^{\infty}\right|_{F_{s}^{2}}$.

Corollary 2.7. Let $\mathcal{M} \subseteq B(\mathcal{K})$ with $I_{\mathcal{H}} \in \mathcal{M}=\mathcal{M}^{*}$ and let $\mathcal{E} \subseteq F_{s}^{2}$ be an invariant subspace under each $B_{i}^{*}:=\left.S_{i}^{*}\right|_{F_{s}^{2}}, i=1, \ldots, n$. If $X \in B(\mathcal{E} \otimes \mathcal{K})$ commutes with $\left.P_{\mathcal{E} \otimes \mathcal{K}}\left(\mathcal{W}_{n}^{\infty} \otimes \mathcal{M}\right)\right|_{\mathcal{E} \otimes \mathcal{K}}$, then there exists $g \in \mathcal{W}_{n}^{\infty} \bar{\otimes} \mathcal{M}^{\prime}$ such that

$$
\left.P_{\mathcal{E} \otimes \mathcal{K}} g\right|_{\mathcal{E} \otimes \mathcal{K}}=X, \quad\|g\|=\|X\| .
$$

Moreover, $\left[\left.P_{\mathcal{E} \otimes \mathcal{K}}\left(\mathcal{W}_{n}^{\infty} \otimes \mathcal{M}\right)\right|_{\mathcal{E} \otimes \mathcal{K}}\right]^{\prime}=\left.P_{\mathcal{E} \otimes \mathcal{K}}\left[\mathcal{W}_{n}^{\infty} \bar{\otimes} \mathcal{M}^{\prime}\right]\right|_{\mathcal{E} \otimes \mathcal{K}}$.

Proof. Since $F_{s}^{2}$ is invariant to $S_{i}^{*}, i=1, \ldots, n$, it is easy to see that $\mathcal{E}$ has the same property. Taking into account that $\mathcal{W}_{n}^{\infty}$ is the compression of $F_{n}^{\infty}$ to the symmetric Fock space, we can see that $X$ commutes with $\left.P_{\mathcal{E} \otimes \mathcal{K}}\left(F_{n}^{\infty} \otimes \mathcal{M}\right)\right|_{\mathcal{E} \otimes \mathcal{K}}$. Applying Theorem 2.4, we find $f \in F_{n}^{\infty} \bar{\otimes} \mathcal{M}^{\prime}$ such that $\left.P_{\mathcal{E} \otimes \mathcal{K}}\left(U^{*} \otimes I\right) f(U \otimes I)\right|_{\mathcal{E} \otimes \mathcal{K}}=X$ and $\|X\|=\|f\|$. Hence, $\left.P_{\mathcal{E} \otimes \mathcal{K}} f\right|_{\mathcal{E} \otimes \mathcal{K}}=X$, and, if we set $g:=\left.P_{F_{s}^{2} \otimes \mathcal{K}} f\right|_{F_{s}^{2} \otimes \mathcal{K}}$, then $\left.P_{\mathcal{E} \otimes \mathcal{K}} g\right|_{\mathcal{E} \otimes \mathcal{K}}=X$ and $\|X\| \leqslant\|g\| \leqslant\|f\|=\|X\|$. This shows that $\|X\|=\|g\|$, and the proof is complete.

Let $\mathcal{M}$ be a von Neumann algebra acting on a Hilbert space $\mathcal{K}$. According to the non-commutative von Neumann inequality, if $\phi \in F_{n}^{\infty} \bar{\otimes} \mathcal{M}$, then the map $\lambda \mapsto \phi(\lambda)$ is in $H^{\infty}\left(\mathbb{B}_{n}\right) \bar{\otimes} \mathcal{M}$, where $\mathbb{B}_{n}:=\left\{z \in \mathbb{C}^{n}:|z|<1\right\}$. A consequence of Theorem 2.4 is the following Nevanlinna-Pick-type interpolation problem for the algebra $F_{n}^{\infty} \bar{\otimes} \mathcal{M}$.

Corollary 2.8. Let $\lambda_{1}, \ldots, \lambda_{k}$ be $k$ distinct points in $\mathbb{B}_{n}$ and let $W_{1}, \ldots, W_{k}$ be in the unit ball of a von Neumann algebra $\mathcal{M}$ acting on a Hilbert space $\mathcal{K}$. Then there exists $\Phi \in F_{n}^{\infty} \bar{\otimes} \mathcal{M}$, such that $\|\Phi\| \leqslant 1$ and $\Phi\left(\lambda_{j}\right)=W_{j}, j=1,2, \ldots, k$, if and only if the operator matrix

$$
\left[\frac{I_{\mathcal{K}}-W_{j} W_{i}^{*}}{1-\left\langle\lambda_{j}, \lambda_{i}\right\rangle}\right]_{i, j=1,2, \ldots, k}
$$

is positive definite.

Proof. Construct $\Psi \in R_{n}^{\infty} \bar{\otimes} \mathcal{M}$ such that $\Psi\left(\lambda_{j}\right)=W_{j}, j=1,2, \ldots, k$. Define $X:=$ $\left.P_{\mathcal{N} \otimes \mathcal{K}} \Psi\right|_{\mathcal{N} \otimes \mathcal{K}}$, with $\mathcal{N}$ as defined in Theorem 2.4 from [3], and apply Corollary 2.5. The rest of the proof is similar to [3, Theorem 2.4].

Let $\mathcal{P}_{m}$ be the set of all polynomials in $F^{2}\left(H_{n}\right)$ of degree less than or equal to $m$, and let $\mathcal{P}_{m}^{\infty}:=\left\{p\left(S_{1}, \ldots, S_{n}\right): p \in \mathcal{P}_{m}\right\}$. Let $J_{>m}^{\infty}$ be the WOT-closed two-sided ideal of $F_{n}^{\infty}$ generated by $\left\{S_{\alpha}: \alpha \in \mathbb{F}_{n}^{+},|\alpha|=m+1\right\}$. Another consequence of Theorem 2.4 (when $\left.\mathcal{N}:=\mathcal{P}_{m}\right)$ is the following result that generalizes the non-commutative Carathéodory interpolation problem [19, Corollary 4.4$]$ to our setting. 
Corollary 2.9. Let $\mathcal{M}$ be a von Neumann algebra acting on a Hilbert space $\mathcal{K}$, and let $f \in \mathcal{P}_{m}^{\infty} \otimes \mathcal{M}$. Then

$$
\operatorname{dist}\left(f, J_{>m}^{\infty} \bar{\otimes} \mathcal{M}\right)=\left\|\left.P_{\mathcal{P}_{m} \otimes \mathcal{K}} f\right|_{\mathcal{P}_{m} \otimes \mathcal{K}}\right\| .
$$

\section{Non-commutative Poisson transforms and functional calculus}

A variant of the non-commutative Poisson transform (see $[\mathbf{2 2}, \S 8]$ ) is used to extend the von Neumann-type inequalities from $[\mathbf{4}, \mathbf{1 2}, \mathbf{1 6}, \mathbf{2 0}, \mathbf{2 2}, \mathbf{2 6}]$. This will lead to a generalization of the functional calculus for contractive sequences of class $C_{0}$ (see [18]), which also extends the Sz.-Nagy-Foiaş $H^{\infty}$-functional calculus for $C_{\text {.0 }}$ (see [25]).

A sequence $\mathcal{T}:=\left[T_{1}, \ldots, T_{n}\right], T_{i} \in B(\mathcal{H})$, is called $C_{0}$-row contraction if $\mathcal{T}$ is a row contraction and

$$
\text { SOT- } \lim _{k \rightarrow \infty} \sum_{\alpha \in \mathbb{F}_{n}^{+},|\alpha|=k} T_{\alpha} T_{\alpha}^{*}=0 .
$$

For example, if $\sum_{i=1}^{n} T_{i} T_{i}^{*} \leqslant a I_{\mathcal{H}}$ for some $a<1$, then $\mathcal{T}$ is a $C_{0}$-row contraction. Suppose that $\mathcal{T}$ is a $C_{0}$-row contraction and let $\Delta:=I_{\mathcal{H}}-\sum_{i=1}^{n} T_{i} T_{i}^{*}$. The Poisson kernel $K\left(\left\{T_{i}\right\}\right)$ associated to $\mathcal{T}$ is the linear map

$$
K\left(\left\{T_{i}\right\}\right): \mathcal{H} \rightarrow F^{2}\left(H_{n}\right) \otimes \mathcal{H} \quad \text { defined by } K\left(\left\{T_{i}\right\}\right) h:=\sum_{\alpha \in \mathbb{F}_{n}^{+}} e_{\alpha} \otimes \Delta^{1 / 2} T_{\alpha}^{*} h .
$$

Since $\sum_{\alpha \in \mathbb{F}_{n}^{+}} T_{\alpha} \Delta T_{\alpha}^{*}=I_{\mathcal{H}}$, the Poisson kernel is an isometry. Notice that

$$
\left(S_{\alpha}^{*} \otimes C^{*}\right) K\left(\left\{T_{i}\right\}\right) h=K\left(\left\{T_{i}\right\}\right) T_{\alpha}^{*} C^{*} h
$$

for any $\alpha \in \mathbb{F}_{n}^{+}$, and $C \in\left\{T_{1}, \ldots, T_{n}, \Delta\right\}^{\prime}$ (the prime stands for the commutant). Hence,

$$
K\left(\left\{T_{i}\right\}\right)^{*}\left(S_{\alpha} S_{\beta}^{*} \otimes A C^{*}\right) K\left(\left\{T_{i}\right\}\right)=A T_{\alpha} T_{\beta}^{*} C^{*}
$$

for any $\alpha, \beta \in \mathbb{F}_{n}^{+}$and $A, C \in\left\{T_{1}, \ldots, T_{n}, \Delta\right\}^{\prime}$. We define the Poisson transform associated with $\mathcal{T}$ to be the map

$$
\Psi_{\mathcal{T}}: B\left(F^{2}\left(H_{n}\right) \otimes \mathcal{H}\right) \rightarrow B(\mathcal{H}), \quad \Psi_{\mathcal{T}}(X):=K\left(\left\{T_{i}\right\}\right)^{*} X K\left(\left\{T_{i}\right\}\right) .
$$

Notice that $\Psi_{\mathcal{T}}$ is unital, completely contractive, $w^{*}$-continuous, and

$$
\Psi_{T}\left(S_{\alpha} S_{\beta}^{*} \otimes A C^{*}\right)=A T_{\alpha} T_{\beta}^{*} C^{*}
$$

for any $\alpha, \beta \in \mathbb{F}_{n}^{+}$, and $A, C \in\left\{T_{1}, \ldots, T_{n}, \Delta\right\}^{\prime}$.

Proposition 3.1. Let $\mathcal{T}:=\left[T_{1}, \ldots, T_{n}\right]$ be a $C_{0}$-row contraction and let $K\left(\left\{T_{i}\right\}\right)$ be its Poisson kernel. If $\mathcal{N}$ is a subspace of $F^{2}\left(H_{n}\right)$ invariant under $S_{1}^{*}, \ldots, S_{n}^{*}$ and $K\left(\left\{T_{i}\right\}\right)$ takes values in $\mathcal{N} \otimes \mathcal{H}$, then the map $\Phi: B(\mathcal{N} \otimes \mathcal{H}) \rightarrow B(\mathcal{H})$ defined by

$$
\Phi(X)=K\left(\left\{T_{i}\right\}\right)^{*} X K\left(\left\{T_{i}\right\}\right)
$$

is unital, completely contractive, $w^{*}$-continuous, and

$$
\Phi\left(B_{\alpha} B_{\beta}^{*} \otimes A C^{*}\right)=A T_{\alpha} T_{\beta}^{*} C^{*},
$$

for every $\alpha, \beta \in \mathbb{F}_{n}^{+}, A, C \in\left\{T_{1}, \ldots, T_{n}, \Delta\right\}^{\prime}$, where $B_{i}:=\left.P_{\mathcal{N}} S_{i}\right|_{\mathcal{N}}, i=1, \ldots, n$. 
Proof. Since $\mathcal{N} \subseteq F^{2}\left(H_{n}\right)$ is an invariant subspace of $S_{1}^{*}, \ldots, S_{n}^{*}$, it is clear that $\left.P_{\mathcal{N}} S_{\alpha} S_{\beta}^{*}\right|_{\mathcal{N}}=B_{\alpha} B_{\beta}^{*}$ for every $\alpha, \beta \in \mathbb{F}_{n}^{+}$. Since $K\left(\left\{T_{i}\right\}\right)=\left(P_{\mathcal{N}} \otimes I_{\mathcal{H}}\right) K\left(\left\{T_{i}\right\}\right)$, Relation (3.1) implies that

$$
\begin{aligned}
A T_{\alpha} T_{\beta}^{*} C^{*} & =K\left(\left\{T_{i}\right\}\right)^{*}\left(S_{\alpha} S_{\beta}^{*} \otimes A C^{*}\right) K\left(\left\{T_{i}\right\}\right) \\
& =K\left(\left\{T_{i}\right\}\right)^{*}\left(P_{\mathcal{N}} \otimes I_{\mathcal{H}}\right)\left(S_{\alpha} S_{\beta}^{*} \otimes A C^{*}\right)\left(P_{\mathcal{N}} \otimes I_{\mathcal{H}}\right) K\left(\left\{T_{i}\right\}\right) \\
& =K\left(\left\{T_{i}\right\}\right)^{*}\left(B_{\alpha} B_{\beta}^{*} \otimes A C^{*}\right) K\left(\left\{T_{i}\right\}\right)
\end{aligned}
$$

for any $\alpha, \beta \in \mathbb{F}_{n}^{+}$. This completes the proof.

Let $C^{*}\left(S_{1}, \ldots, S_{n}\right)$ be the $C^{*}$-algebra generated by $S_{1}, \ldots, S_{n}$, the extension through compacts of the Cuntz algebra $\mathcal{O}_{n}$ (see [5]). The following result is a generalization of Theorems 8.1 and 9.2 from [22], and Theorem 6.2 from [4].

Theorem 3.2. Let $\mathcal{T}:=\left[T_{1}, \ldots, T_{n}\right]$ be a contractive sequence of operators with $T_{i} \in B(\mathcal{H})$. Then there exists a completely contractive linear map

$$
\Phi_{\mathcal{T}}: C^{*}\left(S_{1}, \ldots, S_{n}\right) \otimes C^{*}\left(T_{1}, \ldots T_{n}\right)^{\prime} \rightarrow B(\mathcal{H}),
$$

such that $\Phi_{\mathcal{T}}\left(S_{\alpha} S_{\beta}^{*} \otimes A\right)=A T_{\alpha} T_{\beta}^{*}$, for any $\alpha, \beta \in \mathbb{F}_{n}^{+}$and $A \in C^{*}\left(T_{1}, \ldots T_{n}\right)^{\prime}$. Moreover, if $T_{1}, \ldots, T_{n}$ are commuting, then the result remains true if one replaces $S_{i}$ by $B_{i}:=$ $\left.P_{F_{s}^{2}\left(H_{n}\right)} S_{i}\right|_{F_{s}^{2}\left(H_{n}\right)}, i=1, \ldots, n$.

Proof. For each $0<r<1$, let $K_{r}\left(\left\{T_{i}\right\}\right)$ be the Poisson kernel associated to $\left[r T_{1}, \ldots, r T_{n}\right]$. Define the linear map

$$
\Phi_{\mathcal{T}}: C^{*}\left(S_{1}, \ldots, S_{n}\right) \otimes C^{*}\left(T_{1}, \ldots T_{n}\right)^{\prime} \rightarrow B(\mathcal{H})
$$

by $\Phi_{\mathcal{T}}(f)=\lim _{r \rightarrow 1} K_{r}\left(\left\{T_{i}\right\}\right)^{*} f K_{r}\left(\left\{T_{i}\right\}\right)$. Using Relation (3.1), one can see that the limit exists in the uniform topology of $B(\mathcal{H})$ and $\Phi_{\mathcal{T}}$ is unital, completely contractive, and $\Phi_{\mathcal{T}}\left(S_{\alpha} S_{\beta}^{*} \otimes C\right)=C T_{\alpha} T_{\beta}^{*}$ for every $\alpha, \beta \in \mathbb{F}_{n}^{+}$and $C \in C^{*}\left(T_{1}, \ldots, T_{n}\right)^{\prime}$. The map $\Phi_{\mathcal{T}}$ is called the Poisson transform associated with $\mathcal{T}$.

Assume now that $T_{i} T_{j}=T_{j} T_{i}$ for any $i, j \in\{1, \ldots, n\}$. In this case, the Poisson kernel $K_{r}\left(\left\{T_{i}\right\}\right)$ takes values in $F_{s}^{2}\left(H_{n}\right) \otimes \mathcal{H}$ for every $0<r<1$, where $F_{s}^{2}\left(H_{n}\right)$ is the symmetric Fock space. As in the proof of Proposition 3.1, we deduce that

$$
K_{r}\left(\left\{T_{i}\right\}\right)^{*}\left(B_{\alpha} B_{\beta} \otimes C\right) K_{r}\left(\left\{T_{i}\right\}\right)=r^{|\alpha|+|\beta|} C T_{\alpha} T_{\beta}^{*} .
$$

Since $K_{r}\left(\left\{T_{i}\right\}\right), 0<r<1$, is an isometry, the map $B_{\alpha} B_{\beta}^{*} \otimes C \mapsto C T_{\alpha} T_{\beta}^{*}$, defined on the span of $\left\{B_{\alpha} B_{\beta}^{*} \otimes C: \alpha, \beta \in \mathbb{F}_{n}^{+}, C \in C^{*}\left(T_{1}, \ldots, T_{n}\right)^{\prime}\right\}$, is completely contractive. Therefore, it can be extended to a unital, completely contractive map $\Phi$ : $C^{*}\left(B_{1}, \ldots, B_{n}\right) \otimes C^{*}\left(T_{1}, \ldots T_{n}\right)^{\prime} \rightarrow B(\mathcal{H})$ satisfying $\Phi\left(B_{\alpha} B_{\beta}^{*} \otimes C\right)=C T_{\alpha} T_{\beta}^{*}$ for all $\alpha, \beta \in \mathbb{F}_{n}^{+}$and $C \in C^{*}\left(T_{1}, \ldots, T_{n}\right)^{\prime}$.

Notice that, for any $A, C \in\left\{T_{1}, \ldots, T_{n}, \Delta\right\}^{\prime}, \alpha, \beta \in \mathbb{F}_{n}^{+}$, and $0<r<1$, we have

$$
K_{r}\left(\left\{T_{i}\right\}\right)^{*}\left(S_{\alpha} S_{\beta}^{*} \otimes A C^{*}\right) K_{r}\left(\left\{T_{i}\right\}\right)=r^{|\alpha|+|\beta|} A T_{\alpha} T_{\beta}^{*} C^{*} .
$$

This relation can be used to prove the following result. 
Proposition 3.3. If $\mathcal{T}:=\left[T_{1}, \ldots, T_{n}\right]$ is a row contraction, then

$$
\left\|\sum_{\text {finite }} A_{(\alpha)} T_{\alpha} T_{\beta}^{*} C_{(\beta)}^{*}\right\| \leqslant\left\|\sum_{\text {finite }} S_{\alpha} S_{\beta}^{*} \otimes A_{(\alpha)} C_{(\beta)}^{*}\right\|
$$

for any $A_{(\alpha)}, C_{(\beta)} \in\left\{T_{1}, \ldots, T_{n}, \Delta\right\}^{\prime}$. Moreover, if $T_{1}, \ldots, T_{n}$ are commuting, then one can replace $S_{i}$ by $B_{i}$.

Notice that, in the particular case when $n=1$, we obtain an extension of the ArvesonParrott version of the von Neumann inequality (see [12]). Let $\mathcal{A}_{n}$ be the non-commutative disc algebra and let $\mathcal{A}_{n}^{s}$ be the norm-closed algebra generated by $B_{i}, i=1, \ldots, n$, and the identity on $F_{s}^{2}$.

Corollary 3.4. Let $T_{i} \in B(\mathcal{H}), i=1, \ldots, n$, such that $T_{1} T_{1}^{*}+\cdots+T_{n} T_{n}^{*}=I_{\mathcal{H}}$, then there exists a completely contractive linear map

$$
\Phi: \mathcal{A}_{n} \otimes\left\{T_{1}, \ldots, T_{n}\right\}^{\prime} \rightarrow B(\mathcal{H})
$$

such that $\Phi\left(S_{\alpha} \otimes A\right)=A T_{\alpha}$, for any $A \in\left\{T_{1}, \ldots, T_{n}\right\}^{\prime}$ and $\alpha \in \mathbb{F}_{n}^{+}$. Moreover, if $T_{1}, \ldots, T_{n}$ are commuting, then one can replace $S_{i}$ by $B_{i}$, and $\mathcal{A}_{n}$ by $\mathcal{A}_{n}^{s}$.

Let us remark that all the results presented so far in this section can be extended to the class of sequences of operators with property $(P)$ (see $[\mathbf{2 2}]$ for terminology). We leave this task to the reader.

According to Lemma 2.2 and Theorem 2.3, every $f \in F_{n}^{\infty} \bar{\otimes}\left\{T_{1}, \ldots, T_{n}, \Delta\right\}^{\prime}$ has a unique Fourier expansion

$$
f \sim \sum_{\alpha \in \mathbb{F}_{n}^{+}} S_{\alpha} \otimes W_{(\alpha)}
$$

with $W_{(\alpha)} \in\left\{T_{1}, \ldots, T_{n}, \Delta\right\}^{\prime}$. For any $0<r<1$, define

$$
f_{r}:=\sum_{\alpha \in \mathbb{F}_{n}^{+}} r^{|\alpha|} S_{\alpha} \otimes W_{(\alpha)}
$$

and

$$
f_{r}\left(T_{1}, \ldots, T_{n}\right):=\sum_{\alpha \in \mathbb{F}_{n}^{+}} r^{|\alpha|} W_{(\alpha)} T_{\alpha} \in B(\mathcal{H}) .
$$

The convergence of this series is uniform. Indeed, using Theorem 3.2, we infer that

$$
\begin{aligned}
\left\|\sum_{|\alpha| \geqslant m} r^{|\alpha|} W_{(\alpha)} T_{\alpha}\right\| & \leqslant\left\|\sum_{|\alpha| \geqslant m} r^{|\alpha|} S_{\alpha} \otimes W_{(\alpha)}\right\| \leqslant \sum_{k \geqslant m} r^{k}\left\|\sum_{|\alpha|=k} S_{\alpha} \otimes W_{(\alpha)}\right\| \\
& \leqslant\left(\sum_{k \geqslant m} r^{k}\right)\left\|\sum_{|\alpha| \geqslant m} W_{(\alpha)}^{*} W_{(\alpha)}\right\|^{1 / 2} .
\end{aligned}
$$

Therefore, $\left\|\sum_{|\alpha| \geqslant m}{ }^{|\alpha|} W_{(\alpha)} T_{\alpha}\right\| \rightarrow 0$ as $m \rightarrow \infty$. The following result is a generalization of the $F_{n}^{\infty}$-functional calculus for $C_{0}$-row contractions. 
Theorem 3.5. Let $f \in F_{n}^{\infty} \bar{\otimes}\left\{T_{1}, \ldots, T_{n}, \Delta\right\}^{\prime}$ and let $\mathcal{T}:=\left[T_{1}, \ldots, T_{n}\right]$ be a $C_{0}$-row contraction. Then $S O T-\lim _{r \rightarrow 1} f_{r}\left(T_{1}, \ldots, T_{n}\right)$ exists and the map

$$
f \mapsto f\left(T_{1}, \ldots, T_{n}\right):=\text { SOT-lim } \lim _{r \rightarrow 1} f_{r}\left(T_{1}, \ldots, T_{n}\right)
$$

is a WOT-continuous completely contractive homomorphism.

Proof. Since $\mathcal{T}$ is a $C_{0}$-row contraction, Relation (3.1) implies that

$$
f_{r}\left(T_{1}, \ldots, T_{n}\right)=K\left(\left\{T_{i}\right\}\right)^{*}\left(\sum_{\alpha \in \mathbb{F}_{n}^{+}} r^{|\alpha|} S_{\alpha} \otimes W_{(\alpha)}\right) K\left(\left\{T_{i}\right\}\right),
$$

where $K\left(\left\{T_{i}\right\}\right)$ is the Poisson kernel associated with $\mathcal{T}$. Since $f=$ SOT-lim $_{r \rightarrow 1} f_{r}$ (see the proof of Theorem 2.3), we deduce that SOT- $\lim _{r \rightarrow 1} f_{r}\left(T_{1}, \ldots, T_{n}\right)$ exists. On the other hand, Relation (3.3) and the non-commutative von Neumann inequality show that $\left\|f_{r}\left(T_{1}, \ldots, T_{n}\right)\right\| \leqslant\left\|f_{r}\right\| \leqslant\|f\|$ for any $0<r<1$. Therefore, $\left\|f\left(T_{1}, \ldots, T_{n}\right)\right\| \leqslant\|f\|$. Since $f\left(T_{1}, \ldots, T_{n}\right)=K\left(\left\{T_{i}\right\}\right)^{*} f K\left(\left\{T_{i}\right\}\right)$, it is clear that $f \mapsto f\left(T_{1}, \ldots, T_{n}\right)$ is WOTcontinuous. This completes the proof.

When $n=1$, Theorem 3.5 is a generalization of the Sz.-Nagy-Foias $H^{\infty}$-functional calculus for $C_{.0}$-contractions. We can also obtain a commutative version of Theorem 3.5. Indeed, if $T_{1}, \ldots, T_{n}$ are commuting, then, according to Proposition 3.1 (when $\mathcal{N}:=F_{s}^{2}$ ), the map $\Phi: B\left(F_{s}^{2} \otimes \mathcal{H}\right) \rightarrow B(\mathcal{H})$ defined by $\Phi(X):=K\left(\left\{T_{i}\right\}\right)^{*} X K\left(\left\{T_{i}\right\}\right)$ is completely contractive and WOT-continuous. Since $\Phi\left(B_{\alpha} \otimes A\right)=A T_{\alpha}$, the map $B_{\alpha} \otimes A \mapsto A T_{\alpha}$ can be extended to a WOT-continuous completely contractive homomorphism from $\mathcal{W}_{n}^{\infty} \bar{\otimes}\left\{T_{1}, \ldots, T_{n}, \Delta\right\}^{\prime}$ to $B(\mathcal{H})$.

Let $J$ be a WOT-closed two-sided ideal of $F_{n}^{\infty}$. Set $\mathcal{N}_{J}:=F^{2}\left(H_{n}\right) \ominus J(1)$, and $B_{i}:=$ $\left.P_{\mathcal{N}_{J}} S_{i}\right|_{N_{J}}, i=1, \ldots, n$. The following theorem generalizes Theorem 3.7 from $[3]$ to our setting.

Theorem 3.6. Let $\mathcal{T}:=\left[T_{1}, \ldots, T_{n}\right]$ be a $C_{0}$-row contraction, and let $J$ be a WOTclosed, two-sided ideal of $F_{n}^{\infty}$ such that $\varphi\left(T_{1}, \ldots, T_{n}\right)=0$ for every $\varphi \in J$, then the linear map $\Phi: B\left(\mathcal{N}_{J} \otimes \mathcal{H}\right) \rightarrow B(\mathcal{H})$ defined by $\Phi(X):=K\left(\left\{T_{i}\right\}\right)^{*} X K\left(\left\{T_{i}\right\}\right)$ is unital, completely contractive, $w^{*}$-continuous and

$$
\Phi\left(B_{\alpha} B_{\beta}^{*} \otimes A C^{*}\right)=A T_{\alpha} T_{\beta}^{*} C^{*},
$$

for every $\alpha, \beta \in \mathbb{F}_{n}^{+}$, and $A, C \in\left\{T_{1}, \ldots, T_{n}, \Delta\right\}^{\prime}$.

Proof. Let $K\left(\left\{T_{i}\right\}\right)$ be the Poisson kernel associated with $\mathcal{T}$. Since $F_{n}^{\infty}$ is the WOTclosed algebra generated by the left creation operators $S_{1}, \ldots, S_{n}$ and the identity, and the $F_{n}^{\infty}$-functional calculus is WOT-continuous, we have

$$
\left\langle K\left(\left\{T_{i}\right\}\right) h, \varphi \otimes k\right\rangle=\left\langle h, \varphi\left(T_{1}, \ldots, T_{n}\right) \Delta^{1 / 2} k\right\rangle=0
$$

for any $h, k \in \mathcal{H}$ and $\varphi \in J$. Hence, we deduce that $K\left(\left\{T_{i}\right\}\right)$ takes values in $\mathcal{N}_{J} \otimes \mathcal{H}$. Now, using Proposition 3.1, the result follows. 
We mention that a characterization of the WOT-closed two-sided ideals of $F_{n}^{\infty}$ was obtained in $[6]$.

Acknowledgements. This work was partly supported by NSF grant DMS-9531954. We thank the referee for helpful comments on the results of this paper.

\section{References}

1. J. Agler and J. E. MCCARthy, Nevanlina-Pick kernels and localization, in Operator Theoretical Methods, Timisoara, 1998, pp. 1-20 (Theta Foundation, Bucharest, 2000).

2. A. Arias and G. Popescu, Factorization and reflexivity on Fock spaces, Integr. Eqns Oper. Theory 23 (1995), 268-286.

3. A. ARIAs and G. Popescu, Non-commutative interpolation and Poisson transforms, Israel J. Math. 115 (2000), 205-234.

4. W. B. ARveson, Subalgebras of $C^{*}$-algebras, III, Multivariable operator theory, Acta Math. 181 (1998), 159-228.

5. J. Cuntz, Simple $C^{*}$-algebras generated by isometries, Commun. Math. Phys. 57 (1977), $173-185$.

6. K. R. Davidson And D. Pitts, Automorphisms and representations of the noncommutative analytic Toeplitz algebras, Math. Annln 311 (1998), 275-303.

7. K. R. DAvidson and D. Pitts, Nevanlinna-Pick interpolation for non-commutative analytic Toeplitz algebras, Integr. Eqns Oper. Theory 31 (1998), 321-337.

8. K. R. DAvidSOn AND D. PitTs, Invariant subspaces and hyper-reflexivity for free semigroup algebras, Proc. Lond. Math. Soc. 78 (1999), 401-430.

9. R. G. Douglas, P. S. Muhly and C. Pearcy, Lifting commuting operators, Michigan Math. J. 15 (1968), 385-395.

10. P. Muhly, B. Solel, Tensor algebras over $C^{*}$-correspondences: representations, dilations, and $C^{*}$-envelopes, J. Funct. Analysis 158 (1998), 389-457.

11. R. Nevanlinna, Über beschränkte Functionen, die in gegebenen Punkten vorgeschribene Werte annehmen, Ann. Acad. Sci. Fenn. A 13 (1919), 7-23.

12. S. Parrott, On a quotient norm and the Sz-Nagy-Foias lifting theorem, J. Funct. Analysis 30 (1978), 311-328.

13. G. PICK, Über die Beschränkungen analytischer Functionen, welche durch vorgegebene Functionswerte bewirkt werden, Math. Annln 77 (1916), 7-23.

14. G. POPESCU, Isometric dilations for infinite sequences of noncommuting operators, Trans. Am. Math. Soc. 316 (1989), 523-536.

15. G. Popescu, Characteristic functions for infinite sequences of noncommuting operators, J. Operat. Theory 22 (1989), 51-71.

16. G. Popescu, Von Neumann inequality for $\left(B(H)^{n}\right)_{1}$, Math. Scand. 68 (1991), 292-304.

17. G. Popescu, On intertwining dilations for sequences of noncommuting operators, $J$. Math. Analysis Appl. 167 (1992), 382-402.

18. G. PopesCu, Functional calculus for noncommuting operators, Michigan Math. J. 42 (1995), 345-356.

19. G. Popescu, Multi-analytic operators on Fock spaces, Math. Annln 303 (1995), 31-46.

20. G. Popescu, Non-commutative disc algebras and their representations, Proc. Am. Math. Soc. 124 (1996), 2137-2148.

21. G. Popescu, Interpolation problems in several variables, J. Math. Analysis Appl. 227 (1998), 227-250.

22. G. Popescu, Poisson transforms on some $C^{*}$-algebras generated by isometries, J. Funct. Analysis 161 (1999), 27-61. 
23. D. Sarason, Generalized interpolation in $H^{\infty}$, Trans. Am. Math. Soc. 127 (1967), 179203.

24. B. Sz.-Nagy and C. Foiaş, Dilation des commutants d'operateurs, C. R. Acad. Sci. Paris Ser. 1266 (1968), 493-495.

25. B. Sz.-NAGY AND C. FoIAS, Harmonic analysis on operators on Hilbert space (NorthHolland, Amsterdam, 1970).

26. J. von NEumanN, Eine Spectraltheorie für allgemeine Operatoren eines unitären Raumes, Math. Nachr. 4 (1951), 258-281. 